\title{
Applying FHIR in an Integrated Health Monitoring System
}

\author{
Barbara Franz ${ }^{1}$, Andreas Schuler ${ }^{1}$, Oliver Krauss ${ }^{1}$ \\ ${ }^{1}$ University of Applied Sciences Upper Austria, School of Informatics, Communication and Media, A-4232 Hagenberg/Austria
}

\begin{abstract}
The continuous monitoring of vital signs has become an important supplement to traditional medical treatment to ensure the success of a therapy. Integrated health monitoring solutions based on existing health standards provide interoperability and enable healthcare providers and patients to exchange and access their data across institutional borders. This paper shows an integrated monitoring solution based on Continua and Integrating the Healthcare Enterprise, which has been tested by more than 130 patients and 14 healthcare institutions. According to user feedback, one recurring problem is the low battery life of smartphones due to high data traffic. Since the recently developed HL7 standard FHIR offers a resource efficient handling of web service connections, a possible approach to extend the monitoring solution to support FHIR.
\end{abstract}

Comparing both solutions using data collected by 68 patients, it can be concluded that there is a significant decrease in data traffic when relying on a RESTful architecture in combination with FHIR. For productive use of the FHIR-based approach shown in this paper, security related concerns have to be taken into account in future work to ensure authenticity and authorization.

\section{Keywords}

Fast Healthcare Interoperability Resources; Integrating the Healthcare Enterprise; Continua Health Alliance; Telemonitoring; Electronic Health Record

\section{Correspondence to:}

\section{Barbara Franz}

University of Applied Sciences Upper Austria School of Informatics, Communication and Media

Address: Softwarepark 11, 4232 Hagenberg/Austria

E-mail: Barbara.Franz@fh-hagenberg.at

\section{EJBI 2015; 11(2):en51-en56}

received: October 31, 2014

accepted: January 8, 2015

published: January 20, 2015

\section{Introduction}

People suffering from chronical diseases have to measure their vital signs like blood pressure, heart frequency, blood sugar and weight regularly. Especially in rehabilitation, for example after cardiologic surgery, the continuous monitoring of vital signs has become an important supplement to traditional medical treatment to ensure the success of a therapy [1]. Most available personal health monitoring solutions are isolated applications, which provide results only to the person conducting the measurement, i.e. the patient, or rely on proprietary protocols concerning the exchange and access to measured data. Thus, healthcare providers often lack access to the monitoring results. Integrated health monitoring solutions based on existing health standards provide interoperability and enable healthcare providers and patients to exchange and access their data across institutional borders 2], see Figure 1. This paper shows an integrated health monitoring solution, which has been developed for several use cases, e.g.:
- Monitoring of patients in cardiologic rehabilitation to examine, whether the monitoring of vital signs can support the rehabilitation process, while enhancing the compliance of patients.

- Monitoring of patients with defined medical indications in hospital environments

- Monitoring of elderly people suffering from chronic diseases, especially patients/customers in care centres who regularly measure blood pressure, weight and blood sugar, to find out if telemonitoring supports medical care by providing physicians with detailed information of their patient's health status.

The presented solution has been evaluated in several small test settings over the last five years. From Jan. to Sept. 2014 it has been tested by more than 130 patients and 14 healthcare institutions consisting of medical practitioners, hospitals, rehabilitation centres, nursing homes as well as care centers. It is still in development and is being constantly refined. 


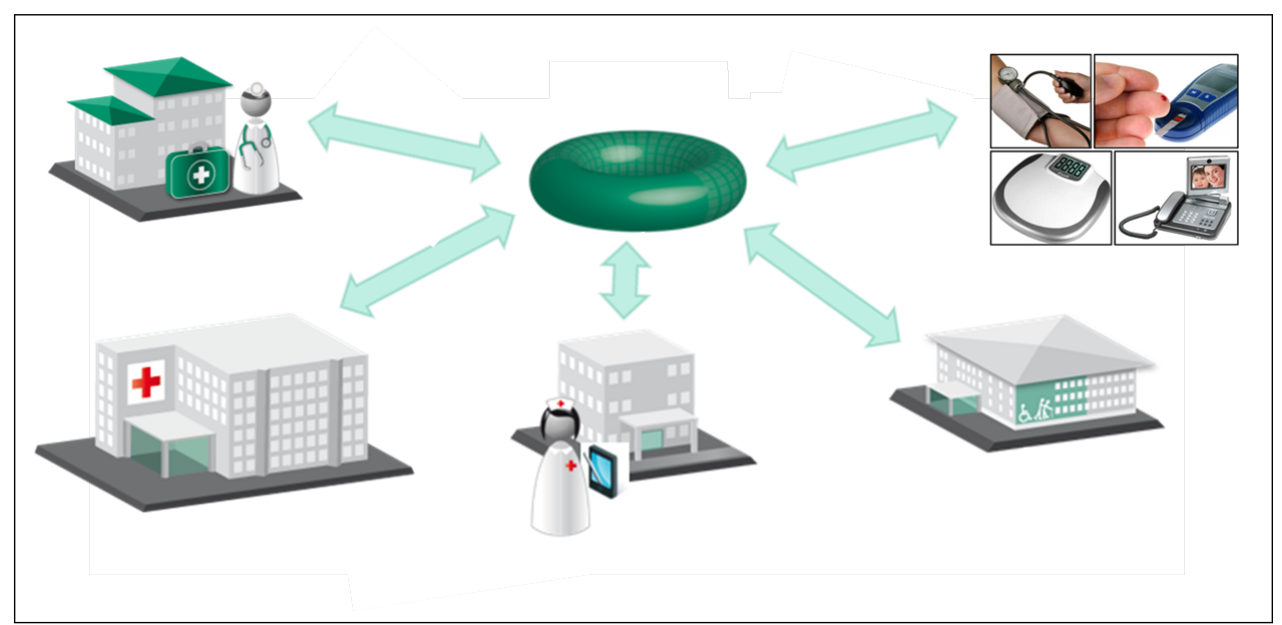

Figure 1: Integrated health monitoring solution used by medical practitioners, hospitals, rehabilitation centres, nursing homes and patients.

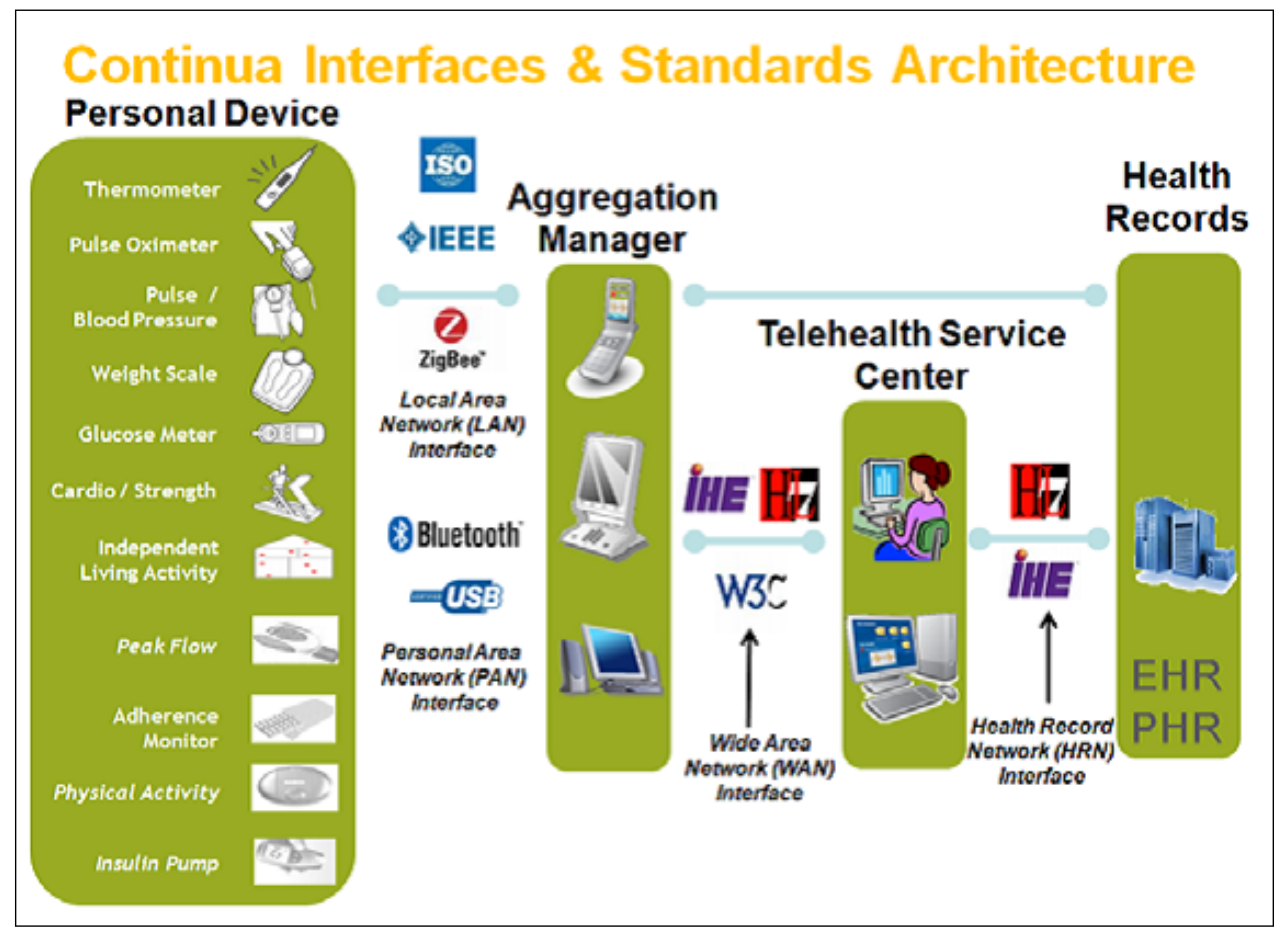

Figure 2: Continua Interfaces and Architecture define use of IHE and HL7 for interoperability [6].

According to user feedback, one recurring problem is the low battery life of smartphones due to high data traffic. Since the recently developed Health Level 7 (HL7) standard Fast Healthcare Interoperability Resources (FHIR) 3] offers a resource efficient handling of web service connections while still preserving the stipulated interoperability through the use of standards connected to HL7, the application of FHIR in context of an integrated health monitoring solution seems worth investigating. Hence, this paper shows a possible approach to extend a health monitoring system to support FHIR.

\section{Methods}

\subsection{Integrated Health Monitoring System}

Since the health monitoring system has to be developed as a medical device, it is based on guidelines defined by the Continua Health Alliance (Continua), which require the use of Integrating the Healthcare Enterprise (IHE) and HL7 to provide interoperability between medical devices and other healthcare systems (see Figure 22). The exchange of data monitored by patient care devices (PCD) is based on the ISO/IEEE 11073 and implements the IHE Device Enterprise Communication (DEC) profile. According to IHE and Continua, a measurement 


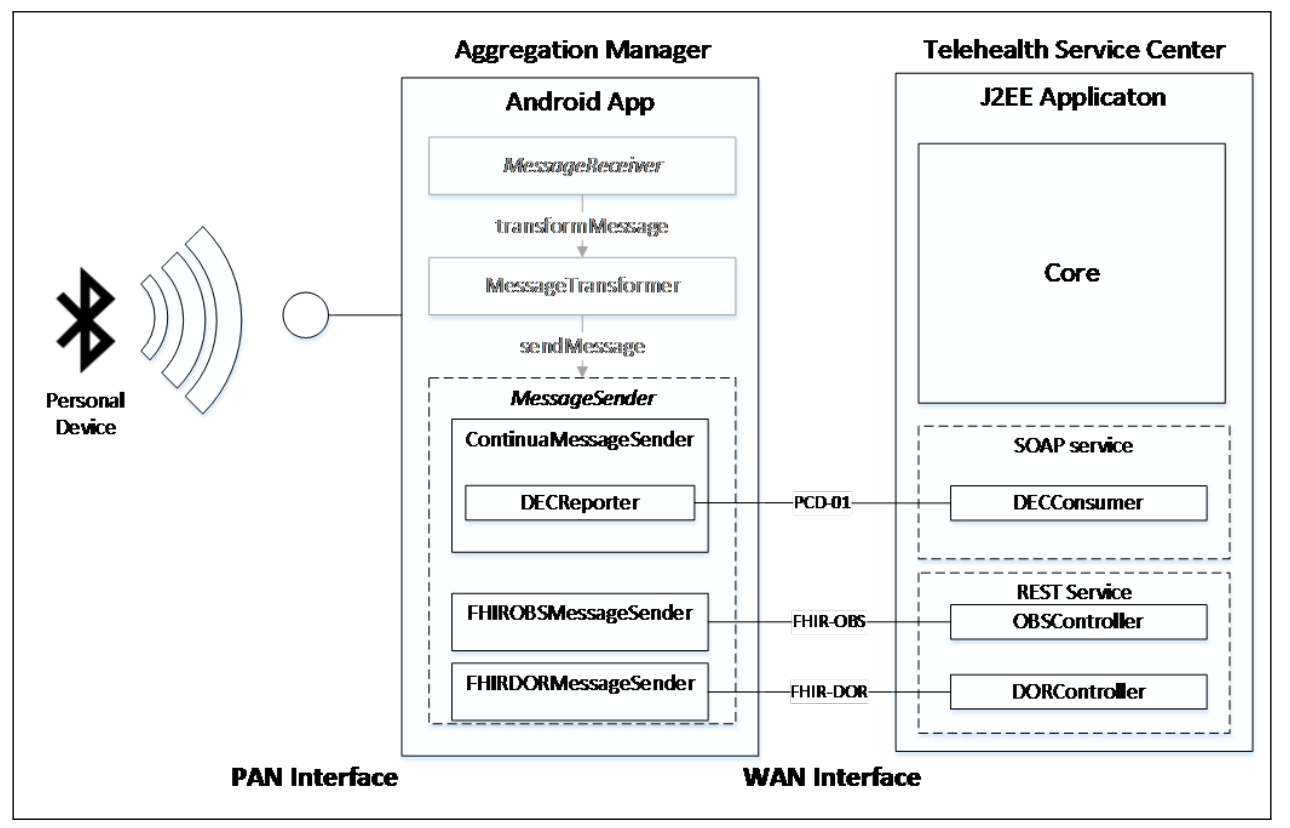

Figure 3: Continua Interfaces and Architecture define use of IHE and HL7 for interoperability [6].

recorded with a telemonitoring solution, is processed as follows [4, 5] (see Figure 2): A personal device, e.g. a scale, sends each measurement to a device, which acts as aggregation manager. The MessageReceiver is responsible for establishing, maintaining and releasing connections to associated personal devices. As a next step, any received data is forwarded to a MessageTransformer that decodes measurements into a human readable format. Finally, the results are transferred using the MessageSender, which acts as DEC Reporter. Hence, it is responsible for, encoding the measurements according to the defined requirements, and sending them to the Telehealth Service Center via a PCD-01 transaction. For transmission, PCD-01 uses a HL7 V2.6 Observation Result Unsolicited (ORU)^R01 message in combination with Simple Object Access Protocol (SOAP). The message contains information on the measurement, the device and the demographic data which enables to establish a link between the information and a patient (realized through extending DEC with Patient Identity Binding, ITI-21 [5]).

As a prerequisite, the patient has to be identified during the measurement, usually done using a unique identifier. Patients using a personalized smartphone, which is linked to the PID, can be assigned directly. To identify patients measuring in a multi-user environment, for example a station in a nursing home, the user is assigned a QR-Code printed on a personal ID-Card.

The Telehealth Service Center acts as DEC Consumer and receives the measurement data. Using Cross Enterprise Document Sharing (XDS), it checks if a Personal Health Monitoring Report (PHMR) has already been registered in the Electronic Health Record (EHR) for the defined patient. The THSC then either creates a new PHMR or it extends the existing document. To avoid large CDA documents, a new version of a PHMR is created every month or depending on a configurable amount of maximum measurements within onereport. Each PHMR document is stored in the EHR, i.e. in an XDS repository. Authorized practicioners and other health personnel can request the PHMR for their patients. The documents are transformed using an extended EXtensible Stylesheet Language Transformations (XSLT) based on the Autrian Electronic Health Record (ELGA) stylesheet.

\subsection{Applying FHIR}

To apply FHIR to the solution described above, it was necessary to extend the WAN-Interface located between the Aggregation Manager and the Telehealth Service Center (see Figure 2). The HL7 message used in connection with the PCD-01 transaction consists of a patient identification, measured data (e.g. weight) and the unit of measure encoded in a HL7 $\mathrm{ORU}^{\wedge} \mathrm{R} 01$ message. The FHIR resources Observation and DeviceObservationReport were chosen since they are a suitable alternative to the HL7 $\mathrm{ORU}^{\wedge} \mathrm{R} 01$ message in FHIR. Hence, two additional MessageSenders were created, as depicted in Figure 3

1. FHIROBSMessageSender responsible for transferring measured data as FHIR-Observations (FHIROBS) resources

2. FHIRDORMessageSender responsible for the transport of FHIR-DeviceObservationReports (FHIRDOR)

An Observation resource contains measurements and simple assertions made about a patient, devices and other subjects [8]. Devices are administrative resources that track individual devices of all kinds and their location [9]. In order to send multiple observations at once, the FHIR 


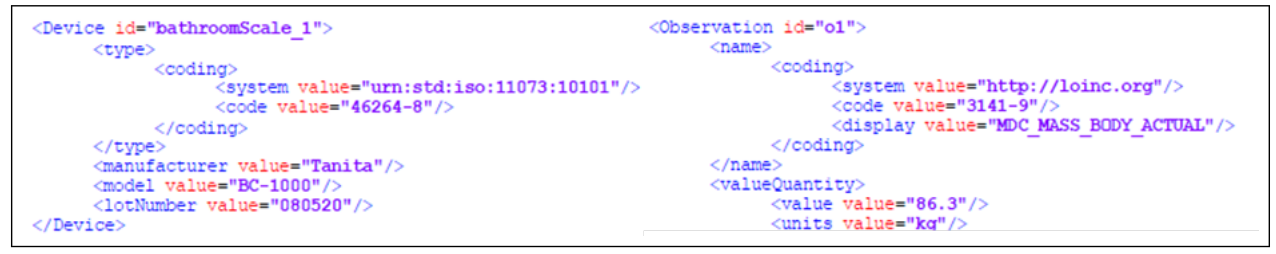

Figure 4: Example for a Device and a simple Observation resource in (XML-format).

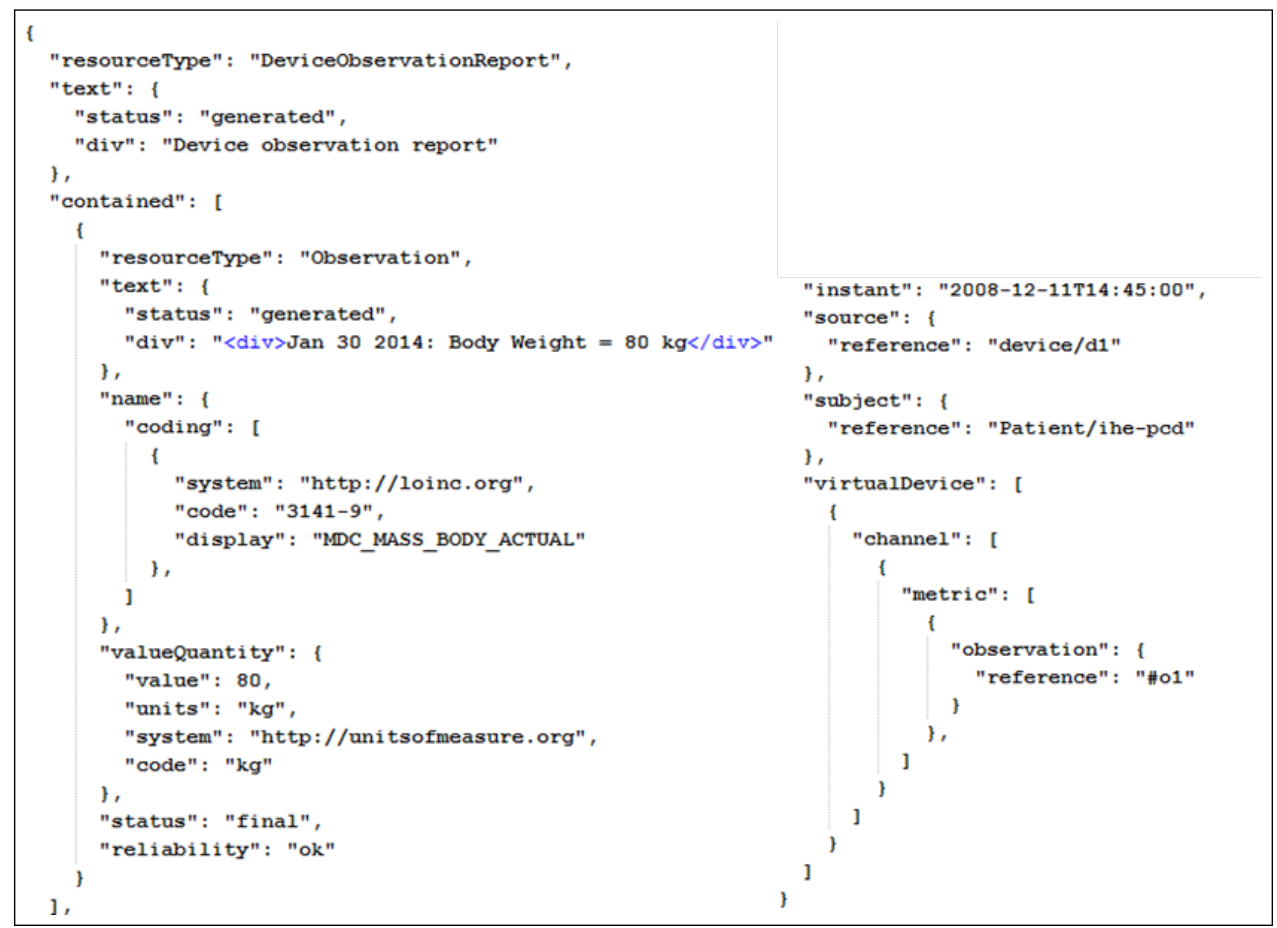

Figure 5: Example of a DeviceObservationReport (JSON-format).

specification offers a bundle concept, which allows grouping of several observations together. Bundles can be used for transactional storing of data [8], e.g. a blood pressure measurement consisting of three observations, the systolic, diastolic and pulse observation. For a simple weight observation, bundling can be omitted (see Figure 4).

The resource DeviceObservationReport describes a set of observations produced by a device at a certain point in time (i.e. the measured data). This resource is based on ISO 11073 and only used for devices which report data 9]. Each DeviceObservationReport contains the measurement, a reference to the patient, the device used for the measurement and a list of observations supplied by the device [8], see Figure 5

The FHIR-based approach was evaluated using data generated during the evaluation of the Continua-based monitoring system. The data collected from 68 patients monitoring their weight for the duration of 5 months, was used to generate the FHIR resources. Only measurements which have been processed by the system successfully were taken into account and used to simulate the extended approach based on real-world user behavior and patient data.

\section{Results}

Conducted surveys, which are described in Table 1 showed, that users (age 29-89) claimed to be pleased or very pleased with using the system. Especially the graphical overview of the measured data (see Figure 6) was considered helpful and important. Particular problems concerning the hardware could be identified. They include poor battery life of the smartphones due to high data traffic, problems using the touch screen of the smartphone and problems in using the measurement devices.

Table 2 shows the average amount of data contributed by PCD-01, FHIR-DOR and FHIR-OBS for weight scale devices over an evaluation period of five months for PCD01 (the Continua compliant system), FHIR-OBS and FHIR-DOR. The data traffic using PCD-01 is 3.2 times higher compared to FHIR-OBS and 2.6 times higher compared to FHIR-DOR.

\section{Conclusion and Outlook}

The presented method shows a possible extension of an integrated interoperable health monitoring system using 
Table 1: Overview over conducted surveys.

\begin{tabular}{|c|c|c|}
\hline USE CASE 1 & USE CASE 2 & USE CASE 3 \\
\hline Cardiologic Reha & Monitoring via hospital & Monitoring of elderly people \\
\hline Patients were monitored over $2.924 \mathrm{~d}$ & Patients were monitored over $1.582 \mathrm{~d}$ & Patients were monitored over $1.726 \mathrm{~d}$ \\
\hline Evaluation during the project & Evaluation during the project & Evaluation during the project \\
\hline Telephone-interviews $(\mathrm{N}=7)$ & Telephone-interviews $(\mathrm{N}=7)$ & $\begin{array}{l}\text { Tel/personal interviews/discussions in } \\
\text { the user environment }(\mathrm{N}=11)\end{array}$ \\
\hline Summative evaluation & Summative evaluation & Summative evaluation \\
\hline Questionnaire for patients $(\mathrm{N}=20)$ & Questionnaire for patients $(\mathrm{N}=9)$ & $\begin{array}{l}\text { Partially standardized discussions in } \\
\text { the user environment }(\mathrm{N}=11+3 \text { discus- } \\
\text { sion rounds) }\end{array}$ \\
\hline
\end{tabular}

Table 2: Overview over conducted surveys.

\begin{tabular}{|c|c|c|c|c|}
\hline & users & measurements & total data (bytes) & estimated data (bytes) \\
\hline & 68 & 1959 & & \\
\hline PCD-01 & & & 354196 & 944505 \\
\hline FHIR-DOR & & & 138811 & 370159 \\
\hline FHIR-OBS & & & 112290 & 299435 \\
\hline
\end{tabular}

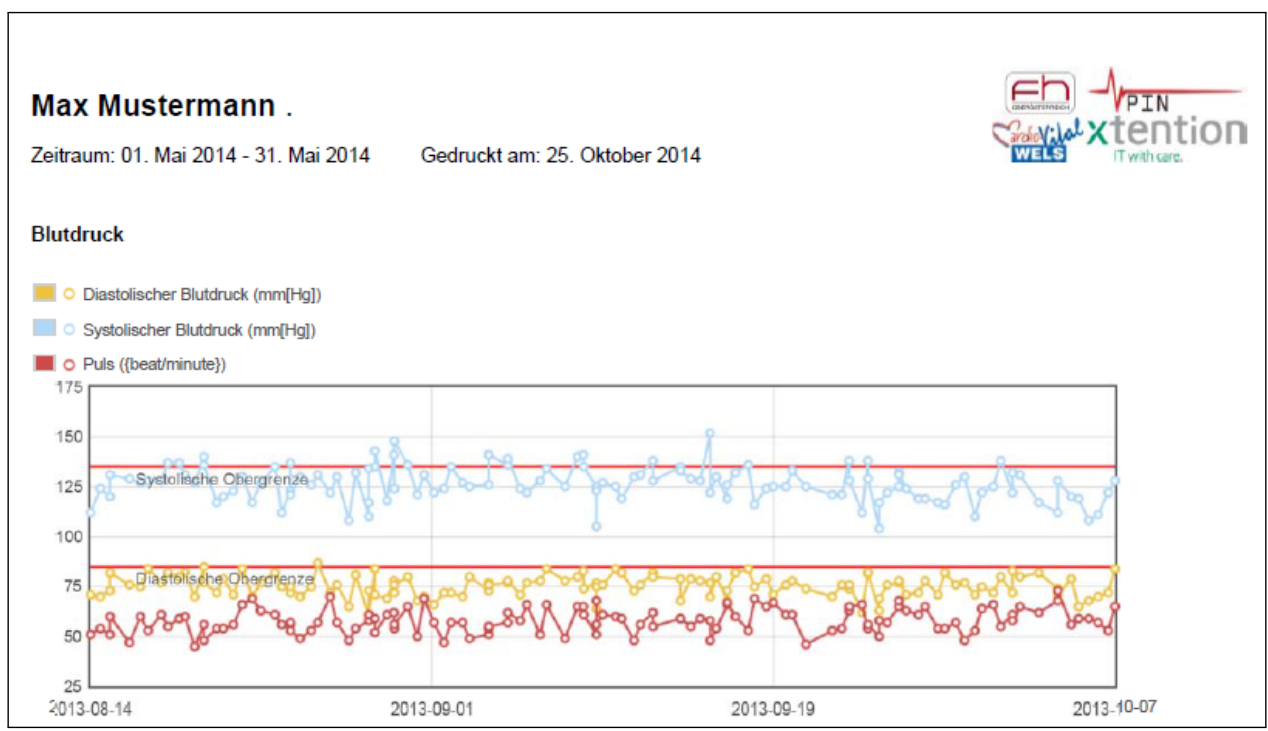

Figure 6: Graphical overview of measured data.

a FHIR-based approach. FHIR uses a RESTful approach concerning efficient exchange of its resources. Thus, it is offering a lightweight alternative to the existing IHE and CHA compliant system, which uses SOAP-based web services. Due to the overhead needed to consume a SOAPbased web service from a mobile device [3] the implementation of a SOAP-based web service client results in increased mobile data traffic and can lead to poor battery life 10. Comparing both solutions using data collected from 68 patients, it can be concluded that there is a significant decrease in data traffic when relying on a RESTful architecture in combination with FHIR. Furthermore due to the decreased amount of data transferred between the Aggregation Manager and the Telehealth Service Center it can be assumed that the battery life of the Smartphones used for transmitting the measurements can be increased, which is confirmed by findings of [3]. This might lessen or eliminate altogether one of the major problems shown during the evaluation of the former solution. Early tests confirm this assumption and show that the extension of battery life seems to provide better user experience and thus acceptance for the patients. Nevertheless, further evaluation of battery life extension is still in progress. Evaluations of the original approach show that such an integrated monitoring system can enhance the personal health competence of a patient. Furthermore, it supports medical personnel by providing a periodic overview of a patient's health status, thus enabling better adjustment of a (medical) therapy. It can also help to detect potential risk situations. Hence, it is necessary that the system is implemented as a medical device and adheres to legal regulations that are in place. To enable productive use of the FHIR-based approach shown in this paper though, security related concerns have to be taken into account to 
ensure authenticity, authorization and authority [1]. Any additional standards from WS-* specifications can be integrated into a CHA (thus IHE)-compliant system, when implementing a SOAP-based approach [4. It remains to be seen in future work if a comparable and sufficient solution for the FHIR-based approach can be found, e.g. a combination of Oauth6 for user authorization with the decentral authentication system OpenID7.6.. Although the proposed solution enables to reduce data footprint on devices with limited resources, it no longer fully complies with the CHA guidelines. However IHE which CHA refers to is currently working on the application of FHIR as stated in [11.

\section{References}

[1] European Commission. Green paper on mobile Health ("mHealth"), version 219 final, Brussels, 2014.

[2] M. Strasser, E. Helm, A. Schuler, B. Franz, H. Mayr, C. David (2012): Telemonitoring für mobile Pflegedienste: Entwicklung von standardkonformen Schnittstellen. eHealth2012 - Health Informatics meets eHealth - von der Wissenschaft zur Anwendung und zurück, Wien, Österreich, pp. 179-184.

[3] Bender, D.; Sartipi, K. (2013): HL7 FHIR: An Agile and RESTful approach to healthcare information exchange, Computer-Based Medical Systems (CBMS), IEEE 26th International Symposium, pp. 326-331.
[4] M. Strasser, E. Helm, A. Schuler, M. Fuschlberger, B. Altendorfer (2012): Mobile Access to Healthcare Monitoring Data for Patients and Medical Personnel - Quality of Life through Quality of Information, Italy.

[5] IHE International, Inc. (2013): IHE Patient Care Device (PCD) Technical Framework, Volume 1, PCD TF1, Integration Profiles, Revision 3.0 - Final Text online resource: http://www.ihe.net/-uploadedFiles/Documents/ PCD/IHE_PCD_TF_Vol1.pdf, last accessed: 29.10.2014.

[6] Continua Health Alliance. The Next Generation of Healthcare: Personal Connected Health \& Wellness, 2014. [cited 2014 Oct 04] online resource: http://www.continuaalliance.org

[7] Lehner, M., Mayr, M., Schmidberger S., Mayr H., Schuler A., Mittermair S.: Forschungsprojekt ELGAMON, Research Report, University of Applied Sciences Upper Austria, 2014.

[8] Chatfield, Chris (1978) The holt-winters forecasting procedure, Applied Statistics 264-279.

[9] HL7 Int. Device ObservationReport - FHIR v.0.0.82, 2014 [cited 2014 Oct 03] online resource: http://www.hl7.org/ implement/standards/fhir/deviceobservationreport.html

[10] Vitali, Fabio; Amoroso, Alessandro; Rocetti, Marco, Marfia, Gustavo (2014): RESTful Services for an Innovtive E-Health Infrastructure: A Real Case Study

[11] IHE International, Inc. (2014): IHE Patient Care Device (PCD) White Paper, HL7 FHIR Device Related Resources, Revision 1.0, online resource: ftp://ftp.ihe.net//Patient_Care_Devices/Profiles/ -FHIR/IHE_PCD_HL7_FHIR_WP_ver_1_THC1.pdf 